EPJ Web of Conferences 66, 03018 (2014)

DOI: $10.1051 /$ epjconf/ 20146603018

(C) Owned by the authors, published by EDP Sciences, 2014

\title{
Retarding friction versus white noise in the description of heavy ion fusion
}

\author{
Maria Chushnyakova ${ }^{1,2 a}$, Igor Gontchar ${ }^{1}$ \\ ${ }^{1}$ Physics and Chemistry Department, Omsk State Transport University, 644046 Omsk, Russia \\ ${ }^{2}$ Physics Department, Omsk State Technical University, 644050 Omsk, Russia
}

\begin{abstract}
We performed modeling of the collision of two spherical nuclei resulting in capture. For this aim the stochastic differential equations are used with the white or colored noise and with the instant or retarding friction, respectively. The dissipative forces are proportional to the squared derivative of the strong nucleus-nucleus interaction potential (SnnP). The SnnP is calculated in the framework of the double folding approach with the density-dependent M3Y $N N$-forces. Calculations performed for ${ }^{28} \mathrm{Si}^{14}{ }^{144} \mathrm{Sm}$ reaction show that accounting for the fluctuations typically reduces the capture cross sections by not more than $10 \%$. In contradistinction, the influence of the memory effects is found resulting in about $20 \%$ enhancement of the cross section.
\end{abstract}

During last three decades significant efforts had been undertaken in modeling the heavy ion capture process resulting at the energies well above the Coulomb barrier [1-5]. However, we did not manage to find out any quantitative systematic study of the influence of accounting for the thermal fluctuations in comparison with the memory effects on the calculated cross sections (CS). We revealed recently that it is possible to describe the modern high accuracy capture data using a classical dissipative trajectory model $[6,7]$. Thus the primary goal of the present work is to study the significance of the fluctuations in comparison with the retarding friction.

In our model we account for the dissipative character of the nucleus-nucleus collision and consider the surface friction mechanism [1,2]. For the potential, we use the double folding approach based on the density dependent M3Y NN-forces with the finite range exchange part [8].

For the sake of simplicity we consider collision of two spherical nuclei accounting for the radial motion only. The collective variable is the dimensionless center-of-mass distance $q=R / R_{P T}$ ( $R$ is the distance between the centers of the projectile $(P)$ and target $(T)$ nuclei and $R_{P T}$ is equal to the sum of the half density radii: $R_{P T}=R_{P}+R_{T}$ ). The equations of motion look as follows:

$$
\begin{array}{cr}
d p / d t=F_{U}+F_{c e n}+\Phi_{D q}+\chi \xi \sqrt{2 D_{q}}, & d q / d t=p / m_{q}, \\
F_{U}=-d U_{t o t} / d q, & F_{c e n}=\hbar^{2} L^{2} /\left(m_{q} q^{3}\right),
\end{array}
$$

\footnotetext{
${ }^{a}$ Corresponding author: maria.chushnyakova@gmail.com
} 


$$
\begin{gathered}
\Phi_{D q}=\int_{0}^{t} F_{D q}(s) \Gamma(t-s) d s, \quad F_{D q}(t)=-p(t) / m_{q} \cdot K_{R}\left[d U_{n}(q(t)) / d q\right]^{2}, \\
\Gamma(t-s)=\tau^{-1} \exp (-|t-s| / \tau), \quad D_{q}=\theta K_{R}\left(d U_{n} / d q\right)^{2}, \\
<\xi(t)>=0, \quad<\xi\left(t_{1}\right) \xi\left(t_{2}\right)>=\Gamma\left(t_{1}-t_{2}\right),
\end{gathered}
$$

Here $p$ stands for the linear momentum; $F_{U}, F_{c e n}$, and $\Phi_{D q}$ are the conservative, centrifugal, and retarding frictional forces, respectively. The latter is represented by the integral (3a) due to the memory effects [9-11]. The memory kernel $\Gamma\left(t_{1}-t_{2}\right)$ (Eq. (4a)) which also plays the role of the random force correlation function (Eq. (5b)) is chosen following Refs. [9-11]. $U_{t o t}(q)$ is the total interaction energy of two ions, it consists of the Coulomb $U_{C}(q)$ and nuclear $U_{n}(q)$ parts; $m_{q}=m_{n} A_{P} A_{T} \cdot R_{P T}^{2}\left(A_{P}+A_{T}\right)^{-1}$ is the inertia parameter; $m_{n}$ is the bare nucleon mass; $K_{R}$ denotes the dissipation strength coefficient. The diffusion coefficient $D_{q}$, the temperature $\theta$, and the friction coefficient $K_{R} \cdot\left(U_{n}^{\prime}\right)^{2}$ are related by the Einstein relation $(4 \mathrm{~b})$. The memory effects are characterized by the correlation time $\tau$ which is expected to be of order of $0.1 \mathrm{zs}$ [11].

According to our experience $[6,7]$, the orbital motion do not influence the calculated CS within $1 \%$ therefore we ignore it in the present work. The quantity $\chi$ in Eq. (1a) takes the values either 0 or 1 allowing to turn off and on the fluctuations. Switching between the instant and retarding friction is made using $\tau=0$ or $\tau>0$.

The double-folding $\operatorname{SnnP} U_{n}(q)$ is described in many papers (see, e.g. [12] where the corresponding code is published). We use here the most elaborated version of this potential [8] with the density dependent and finite range M3Y $N N$ forces. The nuclear matter densities are of crucial importance for our model. In Figure 1 these densities multiplied by $4 \pi r^{2}$ are shown for ${ }^{28} \mathrm{Si}$ and ${ }^{144} \mathrm{Sm}$. The yellow dots represent results of the Hartree-Fock calculations [13]. The lines show the two-parameter Fermi distributions

$$
\rho_{A}(r)=\rho_{0 A}\left\{1+\exp \left[\left(r-R_{A}\right) / a_{A}\right]\right\}^{-1}
$$

calculated with different values of the diffuseness $a_{A}$.
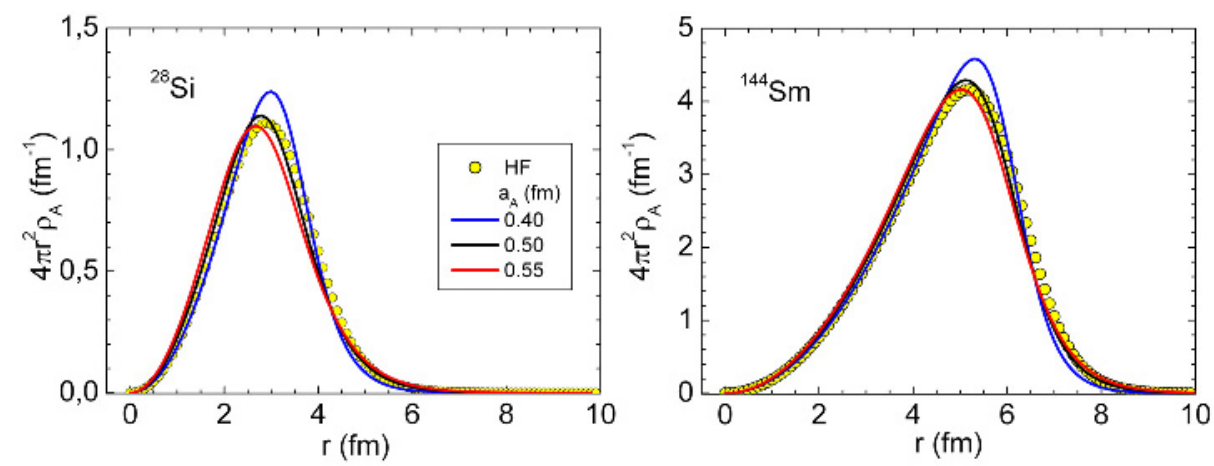

Figure 1. The quantity $4 \pi r^{2} \rho_{A}$ for ${ }^{28} \mathrm{Si}$ (left panel) and ${ }^{144} \mathrm{Sm}$ (right panel). The lines correspond to the densities calculated using Eq. (6) whereas the circles correspond to the Hartree-Fock densities from Refs. [13]. 
Because $\rho_{0 A}$ is extracted from the normalization condition, for the given value of $a_{A}$ the only undefined parameter is the half density radius $R_{A}$. We assume that the nuclear charge radius parameter $R_{q}$ is equal to $R_{A}$ whereas the matter diffuseness is related to the charge diffuseness $a_{q}$ according to Eq. (35) of Ref. [12]. In the first approximation over the small parameter $\pi^{2} a_{q}^{2} R_{q}^{-2}$, the charge radius and diffuseness are related with the root mean square charge radius $R_{m q}$ as follows

$$
R_{q}=\sqrt{5 / 3\left(R_{m q}^{2}-7 \pi^{2} a_{q}^{2} / 5\right)}
$$

We take the values of $R_{m q}$ for both ${ }^{28} \mathrm{Si}$ and ${ }^{144} \mathrm{Sm}$ from Ref. [14]. Figure 1 shows that $a_{A}=0.50 \mathrm{fm}$ provides the best agreement with the Hartree-Fock densities for both nuclei. The calculations below are performed with this value of $a_{A}$. The value of the friction strength is $K_{R}=2.010^{-2} \mathrm{MeV}^{-1} \mathrm{zs}$.

Let us note that ignoring the deformation of the ${ }^{28} \mathrm{Si}$ can only be important for the collsion energies close to the Coulomb barrier and below [15] whereas our model is designed for the higher collision energies.

The capture CS are calculated according to the commonly used formula

$$
\sigma=\frac{\pi \hbar^{2}}{2 m_{R} E_{c . m . ~}} \sum_{L=0}^{L_{\max }}(2 L+1) T_{L}
$$

Obviously the values of the CS should not depend upon the technical parameter $L_{\max }$. Provided the fluctuations are ignored ( $\chi=0$ in Eq. (1a)), the transmission coefficient $T_{L}$ can be equal either 1 if the trajectory corresponding to the particular $L$ is captured, or 0 otherwise. At $E_{c . m .}>U_{B L c}$ all the trajectories with $L$ ranging from 0 up to $L_{c}$ are captured.

In the case $\chi=1$ (the fluctuations are present) $N_{L}=400$ trajectories are simulated for every partial wave; the transmission coefficient is defined as the ratio of the captured trajectories number to $N_{L}$. The total number of the trajectories for every reaction typically ranges between $10^{4}$ and $3 \cdot 10^{4}$.

Results are shown in Figure 2 as the ratios of the fusion CS calculated with different options. The influence of the fluctuations is illustrated by the left panel. It shows that with the instant friction (green circles) the fluctuations reduce the CS in comparison with the deterministic case typically by 5 $10 \%$. This might be expected since due to the fluctuations a trajectory which otherwise would fuse has a chance to be scattered back. In the presence of the retardation ( $\tau=0.2 \mathrm{zs}$, red boxes) this effect is suppressed: the ratio $\sigma_{\mathrm{f} \tau} / \sigma_{\mathrm{d} \tau}$ deviates chaotically from unity by $2-4 \%$. These irregularities are not due to the thermal fluctuations as discussed in details in Appendix of Ref. [6].
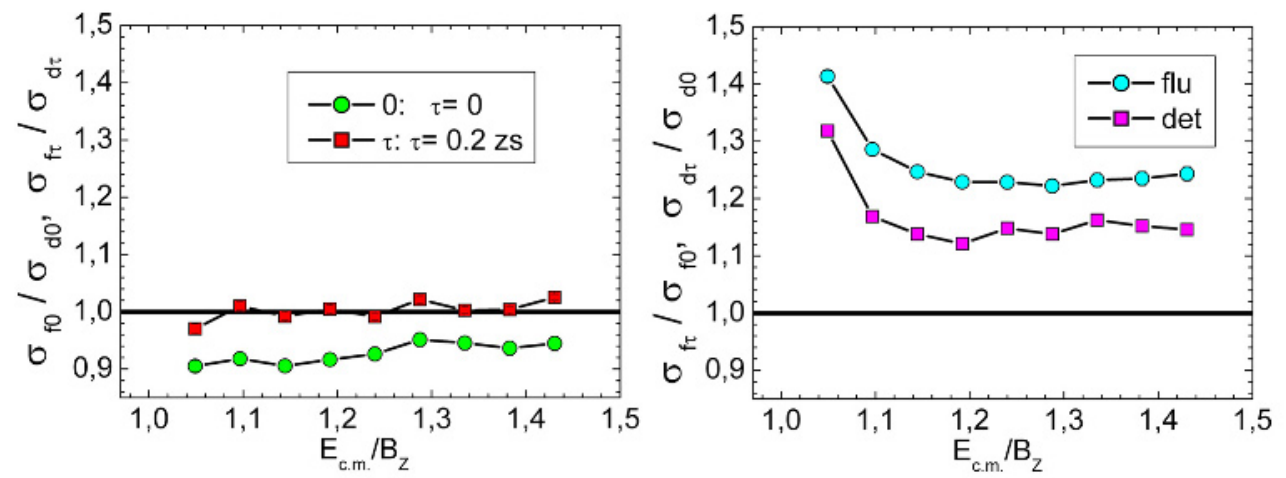

Figure 2. Left panel: the influence of the fluctuations in the case of the white noise (green circles) and of the coloured noise (red boxes). Right panel: the influence of the retardation in the case of the deterministic calculations (magenta boxes) and of the calculations with fluctuations (cyan circles). 
The influence of the retardation is illustrated by the right panel of Figure 2. Both in the absence and in the presence of the fluctuations accounting for the finite $\tau$ elevates the CS by $15 \%$ to $40 \%$. In order to understand this effect it is useful to inspect Figure 3 where the radial dependence of the friction coefficient is presented. For larger values of the coordinate the friction coefficient is significantly smaller because of the derivative of the SnnP. Accounting for the retardation effectively memorizes the lower values of the friction coefficient resulting in the higher values of the fusion CS.

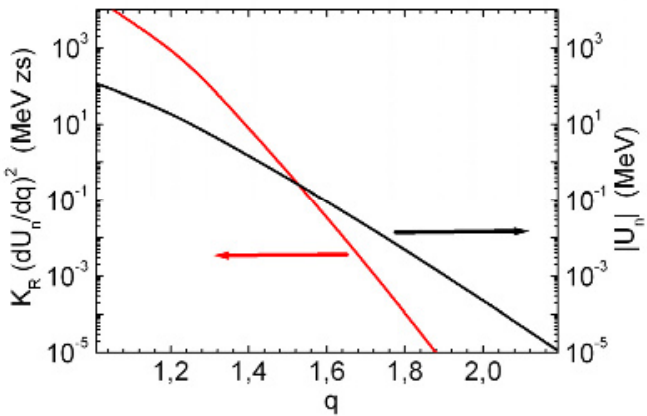

Figure 3. The coordinate dependence of the friction coefficient (left axes, red line) and the absolute value of the SnnP (right axes, black line).

To summarize, in this work we compare the influence of the thermal fluctuations and the retarding friction on the capture CS in the ${ }^{28} \mathrm{Si}+{ }^{144} \mathrm{Sm}$ reaction at the energies above the Coulomb barrier. For this aim we use an upgraded version of the dynamical model [6]. The double folding potential with the density dependent finite range M3Y $N N$-forces plays a crucial role in this model.

It turns out that accounting for the fluctuations results in decreasing the captures CS by less than $10 \%$ with the instant friction. This reduction is compensated by the retardation at $\tau=0.2 \mathrm{zs}$. Including the memory effects results in the increase of the CS by $10-40 \%$ depending on the collision energy and presence (or absence) of the fluctuations. In general the memory effects influence the capture CS stronger than the noise.

It would be interesting to confront the excitation functions resulting from our model with the experimental ones. However, such comparison goes significantly beyond the limited volume of this presentation. Some preliminary results are shown in $[6,7,16]$. We hope to publish systematic comparison with the data for several reactions in near future [17].

\section{Acknowledgments}

The authors wish to thank R. Bhattacharya for providing the Hartree-Fock density distributions. M. V. C. is grateful to the Dmitry Zimin Foundation "Dynasty" and to the organizers of INPC 2013 for financial support.

\section{References}

1. P. Fröbrich, Phys. Rep. 116, 337 (1984)

2. D.H.E. Gross and H. Kalinowski, Phys. Rep. 45, 175 (1978)

3. V. Zagrebaev and W. Greiner, J. Phys. G. 34, 1 (2007)

4. R. A. Kusyakin et al., Phys. Rev. C 85, 034612 (2012)

5. C. Simenel, Eur. Phys. J. A 48, 152 (2012)

6. M.V. Chushnyakova and I.I. Gontchar, Phys. Rev. C 87, 014614 (2013) 
7. M.V. Chushnyakova and I.I. Gontchar, Eur. Phys. J Web of Conf. HIAS-2013 to be published

8. Dao T. Khoa, G.R. Satchler, W. von Oertzen, Phys. Rev. C 56, 954 (1997)

9. D. Boilley, E. Suraud, Y. Abe, S. Ayik, Nucl. Phys. A 556, 67 (1993)

10. Y. Abe, S. Ayik, P.-G. Reinhard, E. Suraud, Phys. Rep. 275, 49 (1996)

11. A.E. Gegechkori, Yu.A. Anischenko et al, Phys. At. Nucl. 71, 2007 (2008)

12. I.I. Gontchar and M. V. Chushnyakova, Comp. Phys. Comm. 181, 168 (2010)

13. R. Bhattacharya, Jour. Mod. Phys. 4, 5B33 (2013) and private communication

14. I. Angeli, At. Dat. Nucl. Dat. Tables 87, 185 (2004)

15. J. O. Newton et al., Phys. Rev. C 70, 024605 (2004).

16. I.I. Gontchar and M. V. Chushnyakova, Jour. Mod. Phys. 4, 5B1 (2013).

17. I.I. Gontchar, R. Bhattacharya, and M.V. Chushnyakova, to be sumbitted to Eur. Phys. J. A. 
